\title{
Uniqueness Results for the Full Frémond Model of Shape Memory Alloys
}

\author{
N. Chemetov
}

\begin{abstract}
In the paper we show two uniqueness results for problems related to the thermomechanical model proposed by Frémond, which describes the structural phase transitions in the shape memory alloys.
\end{abstract}

Keywords: Uniqueness, Frémond model, shape memory alloys

AMS subject classification: $35 \mathrm{M} 20,35 \mathrm{~K} 60,35 \mathrm{R} 35$

\section{Introduction}

We consider the system of partial differential equations given by

$$
\begin{aligned}
& \partial_{t}\left(c_{0} \theta-l \theta_{*} \chi_{1}\right)+\partial_{t}\left(\left(\alpha(\theta)-\theta \alpha^{\prime}(\theta)\right) \chi_{2} \operatorname{div} \mathbf{u}\right)-h \Delta \theta=F+\alpha(\theta) \chi_{2}(\operatorname{div} \mathbf{u})_{t} \\
& \mathbf{u}_{t t}-\operatorname{div}\left(\lambda \operatorname{div} \mathbf{u} \cdot J+2 \mu E(\mathbf{u})+\alpha(\theta) \chi_{2} \cdot J-\nu \Delta(\operatorname{div} \mathbf{u}) \cdot J\right)=\mathbf{G} \\
& k \frac{\partial}{\partial t}\left[\begin{array}{l}
\chi_{1} \\
\chi_{2}
\end{array}\right]+\left[\begin{array}{c}
l\left(\theta-\theta_{*}\right) \\
\alpha(\theta) \operatorname{div} \mathbf{u}
\end{array}\right]+\partial I_{K}\left(\chi_{1}, \chi_{2}\right) \ni\left[\begin{array}{l}
0 \\
0
\end{array}\right]
\end{aligned}
$$

in $Q=\Omega \times(0, T)$, where $\Omega$ is an open bounded subset of $\mathbb{R}^{3}$. The unknowns $\theta, \mathbf{u}, \chi_{1}, \chi_{2}$ have the following physical meaning : $\theta$ is the temperature of the alloy, $\mathbf{u}=\left(u_{1}, u_{2}, u_{3}\right) \in$ $\mathbb{R}^{3}$ is the displacement vector, and $\chi_{1}, \chi_{2}$ are the transformed phase proportions of different phases of the alloy, that have been obtained by the following: Let $\beta_{1}, \beta_{2}$ and $\beta_{3}$ be the volumetric proportions of two martensitic variants and of the austenite, respectively. The side condition for $\beta_{1}, \beta_{2}, \beta_{3} \in[0,1]$ with $\beta_{1}+\beta_{2}+\beta_{3}=1$ can be equivalently rewritten as

$$
\left(\chi_{1}, \chi_{2}\right) \in K=\left\{\left(\gamma_{1}, \gamma_{2}\right) \in \mathbb{R}^{2}:\left|\gamma_{2}\right| \leq \gamma_{1} \leq 1\right\}
$$

where $\chi_{1}$ and $\chi_{2}$ are defined by $\chi_{1}=\beta_{1}+\beta_{2}$ and $\chi_{2}=\beta_{2}-\beta_{1}$. The functions $F$ and $G$ represent the distributed heat sources and the body forces, respectively, $E(\mathbf{u})$ is the linearized strain tensor and $J$ is the identity matrix in $\mathbb{R}^{3}$. For the physical meaning of the positive constants $c_{0}, h, \nu, \lambda, \mu, k, l$ and $\theta_{*}$ we refer to $[3,7]$. The given function

N. Chemetov, Universidade Independente de Lisboa,Departamento de Matemàtica, Av. Marechal Gomes de Costa, Lote 9, 1800 Lisboa - Portugal, and Universidade de Lisboa, Centro de Matemàtica E Aplicacoes Fundamentais, Av. Prof. Gama Pinto 2, 1699 Lisboa - Portugal 
$\alpha$ represents the thermal expansion of the system and is non-negative, vanishing for any temperature larger than a so-called Curie point $\theta_{c}>\theta_{*}$. Finally, $\partial I_{K}$ denotes the subdifferential of the indicator function

$$
I_{K}= \begin{cases}0 & \text { if }\left(\gamma_{1}, \gamma_{2}\right) \in K \\ +\infty & \text { if }\left(\gamma_{1}, \gamma_{2}\right) \notin K\end{cases}
$$

for the triangle $K$ defined in (1.4).

System (1.1) - (1.3) has been proposed by M. Frémond [3, 7] in 1987 for describing thermo-mechanical processes and structural phase transitions (martensite $\Leftrightarrow$ austenite) in shape memory alloys. Later on this system has been studied under different simplifications in many articles (see a review in $[1,2,4,5]$ ). Here we would like to pay our attention to three articles $[1,2,4]$, where system $(1.1)-(1.3)$ has been investigated in a full formulation. In [4] for the one-dimensional case system (1.1) - (1.3) has been taken in the quasi-stationary statement, that is, the inertial term $\mathbf{u}_{t t}$ has been omitted. Due to these assumptions an explicit form for $u_{x}$ has been obtained. This has allowed to write the system just in the terms of the unknowns $\theta, \chi_{1}, \chi_{2}$ and as a consequence to show both existence and uniqueness results. In [2] $\mathrm{P}$. Colli has established an existence result for the quasi-stationary form of system (1.1) - (1.3) already in the multi-dimensional case. But the uniqueness of solution has remained an open question. Also we would like to mention article [1] where an existence result has been obtained for system (1.1) (1.3) without any simplification in one space dimension. The uniqueness result has not been proved.

The main purpose of our article is to show the uniqueness results for these last two problems.

\section{Formulation of results}

2.1 Formulation of the first result. First we recall some notations. Let $(\cdot, \cdot)$ and $\|\cdot\|$ be the scalar product and the norm in $L^{2}(\Omega)$, respectively, and let us denote by $\mathbf{n}$ the outer unit normal to the boundary $\partial \Omega$ and by $\left\{\Gamma_{0}, \Gamma_{N}\right\}$ a partition of $\partial \Omega$ into two subsets such that $\Gamma_{0}$ has a positive surface measure. We set

$$
\begin{aligned}
\mathbf{K}= & \left\{\left(\gamma_{1}, \gamma_{2}\right) \in\left(L^{\infty}(Q)\right)^{2}:\left|\gamma_{2}\right| \leq \gamma_{1} \leq 1 \text { a.e. in } Q\right\} \\
V= & \left\{\mathbf{v} \in\left(H^{1}(\Omega)\right)^{3}: \mathbf{v}=0 \text { on } \Gamma_{0} \text { and } \operatorname{div} \mathbf{v} \in H^{1}(\Omega)\right\} \\
a(\mathbf{v}, \mathbf{w})= & \int_{\Omega}(\lambda \operatorname{div} \mathbf{v d i v} \mathbf{w} \\
& \left.+2 \mu \sum_{i, j=1}^{3} E_{i j}(\mathbf{v}) E_{i j}(\mathbf{w})+\nu \nabla(\operatorname{div} \mathbf{v}) \nabla(\operatorname{div} \mathbf{w})\right) d x \quad(\mathbf{v}, \mathbf{w} \in V)
\end{aligned}
$$

where

$$
E_{i j}(\mathbf{v})=\frac{1}{2}\left(\frac{\partial v_{i}}{\partial x_{j}}+\frac{\partial v_{j}}{\partial x_{i}}\right) \quad(i, j=1, \ldots, 3)
$$


Concerning the data of the problem we suppose

$$
\begin{aligned}
& F \in L^{2}(Q), \quad f \in H^{1}\left(0, T, L^{2}(\partial \Omega)\right) \\
& \mathbf{G} \in H^{1}\left(0, T,\left(L^{2}(\Omega)\right)^{3}\right), \quad \mathbf{g} \in H^{1}\left(0, T,\left(L^{2}\left(\Gamma_{N}\right)\right)^{3}\right) \\
& \theta_{0} \in H^{1}(\Omega), \quad\left(\chi_{1,0}, \chi_{2,0}\right) \in \mathbf{K} \\
& F \geq 0 \text { a.e. in } Q, \quad \theta_{0} \geq 0 \text { a.e. in } \Omega, \quad f \geq 0 \text { a.e. in }(0, T) \times \partial \Omega
\end{aligned}
$$

and the function $\alpha$ is non-negative, vanishing from the Curie point $\theta_{c}>0$,

$$
\begin{aligned}
& \alpha \in C^{2}(\mathbb{R}) \text { such that } \alpha^{\prime}(\xi)=0 \text { for all } \xi \in \mathbb{R} \backslash\left(0, \theta_{c}\right), \\
& c_{\alpha}=\left\|\alpha^{\prime \prime}\right\|_{L^{\infty}\left(0, \theta_{c}\right)} \text { is sufficiently small. }
\end{aligned}
$$

Remark 1. By (2.1.3) (or by (2.2.3), see below) we easily deduce that $\left|\xi \alpha^{\prime \prime}(\xi)\right|,\left|\alpha^{\prime}(\xi)\right| \leq \theta_{c} c_{\alpha} \quad$ and $\quad\left|\xi \alpha^{\prime}(\xi)\right|,|\alpha(\xi)| \leq \theta_{c}^{2} c_{\alpha}$ for all $\xi \in \mathbb{R}$.

Problem (P1). Find

$$
\begin{aligned}
& \theta \in H^{1}\left(0, T, L^{2}(\Omega)\right) \cap C^{0}\left(0, T, H^{1}(\Omega)\right), \theta \geq 0 \text { a.e. in } Q \\
& \mathbf{u}=\left(u_{1}, u_{2}, u_{3}\right) \in H^{1}(0, T, V) \text { with } \operatorname{div} \mathbf{u} \in C^{0}(\bar{Q}) \\
& \chi_{1}, \chi_{2} \in H^{1}\left(0, T, L^{2}(\Omega)\right), \quad\left(\chi_{1}, \chi_{2}\right) \in \mathbf{K}
\end{aligned}
$$

such that

$$
\begin{aligned}
& \left(\partial_{t}\left(c_{0} \theta-l \theta_{*} \chi_{1}\right)+\partial_{t}\left(\left(\alpha(\theta)-\theta \alpha^{\prime}(\theta)\right) \chi_{2} \operatorname{div} \mathbf{u}\right), \phi\right)+h(\nabla \theta, \nabla \phi) \\
& +\eta \int_{\partial \Omega}(\theta-f) \phi d x=\left(F+\alpha(\theta) \chi_{2}(\operatorname{div} \mathbf{u})_{t}, \phi\right) \\
& \quad \text { a.e. in }(0, T), \text { for all } \phi \in H^{1}(\Omega) \\
& \theta(x, 0)=\theta_{0}(x) \quad \text { a.e. in } \Omega \\
& a(\mathbf{u}, \mathbf{v})+\left(\alpha(\theta) \chi_{2}, \operatorname{div} \mathbf{v}\right)=\int_{\Omega} \mathbf{G v} d x+\int_{\Gamma_{N}} \mathbf{g v} d x \\
& \quad \text { a.e. in }(0, T), \mathbf{v} \in V \\
& \sum_{j=1}^{2} k\left(\partial_{t} \chi_{j}, \chi_{j}-\gamma_{j}\right)+l\left(\theta-\theta_{*}, \chi_{1}-\gamma_{1}\right)+\left(\alpha(\theta) \operatorname{div} \mathbf{u}, \chi_{2}-\gamma_{2}\right) \leq 0 \\
& \quad \text { a.e. in } Q, \text { for all }\left(\gamma_{1}, \gamma_{2}\right) \in \mathbf{K} \\
& \left(\chi_{1}, \chi_{2}\right)(x, 0)=\left(\chi_{1,0}, \chi_{2,0}\right)(x) \quad \text { a.e. in } \Omega .
\end{aligned}
$$

Remark 2. Formally equation (2.1.6) is equivalent to equation (1.2) taken in the quasi-stationary case, i.e. without the inertial term $\mathbf{u}_{t t}$, where $\mathbf{u}$ satisfies the boundary conditions

$$
\begin{aligned}
\left(\left(-\nu \cdot \Delta(\operatorname{div} \mathbf{u})+\lambda \operatorname{div} \mathbf{u}+\alpha(\theta) \chi_{2}\right) J+2 \mu E(\mathbf{u}), \mathbf{n}\right) & =\mathbf{g} \text { on } & & \Gamma_{N} \times[0, T] \\
\mathbf{u} & =0 \text { on } & & \Gamma_{0} \times[0, T] \\
\frac{\partial}{\partial \mathbf{n}}(\operatorname{div} \mathbf{u}) & =0 \text { on } & & \partial \Omega \times[0, T]
\end{aligned}
$$


where $J$ is the unit matrix.

Lemma 1 (see P. Colli, M. Frémond and A. Visintin [3]). For any $\theta, \chi_{2} \in C^{0}(0, T$, $\left.L^{2}(\Omega)\right)$ and $\left|\chi_{2}\right| \leq 1$ a.e. in $Q$, there exists one and only one solution $\mathbf{u}$ of equation (2.1.6) such that

$$
\mathbf{u} \in C^{0}(0, T, V) \quad \text { and } \quad \operatorname{div} \mathbf{u} \in C^{0}(\bar{\Omega}) \quad(t \in[0, T])
$$

and there is a constant $\mathrm{B}$ depending on $c_{\alpha}, \theta_{c}, G, g, \Omega, \nu, \lambda$ and $\mu$ such that

$$
\|\operatorname{div} \mathbf{u}(\cdot, t)\|_{C^{0}(\bar{\Omega})} \leq \mathbf{B} \quad(t \in[0, T]) .
$$

Lemma 2 (see P. Colli [2]). Under the above conditions (2.1.2) and for $\alpha$ satisfying condition (2.1.3), if

$$
0<c_{0}-\theta_{c} \cdot c_{\alpha} \cdot \mathbf{B}=\mathbf{A}
$$

and

$$
\left(\theta_{c}\left(\theta_{c}+1\right) c_{\alpha}\right)^{2} \leq \mathbf{A} \cdot\left(\lambda+\frac{2}{3} \mu\right)
$$

then there exists at least one solution of Problem (P1).

Theorem 1. Under the conditions of Lemma 2 Problem (P1) has one and only one solution.

2.2 Formulation of the second result. Let us formulate the second result of this article. Now we consider system (1.1) - (1.3) in the one-dimensional case, i.e. $\Omega=(0,1)$, and use the notations

$$
\begin{aligned}
H_{z}^{2}(\Omega) & =H^{2}(\Omega) \cap H_{0}^{1}(\Omega) \\
H_{z}^{3}(\Omega) & =\left\{v \in H^{3}(\Omega): v(s)=v_{x x}(s)=0 \quad(s=0,1)\right\} \\
\mathbf{K} & =\left\{\left(\gamma_{1}, \gamma_{2}\right) \in\left(L^{\infty}(Q)\right)^{2}:\left|\gamma_{2}\right| \leq \gamma_{1} \leq 1 \text { a.e. in } Q\right\} \\
a(v, w) & =\beta \int_{\Omega} v_{x} w_{x} d x+\nu \int_{\Omega} v_{x x} w_{x x} d x \quad\left(v, w \in H_{z}^{2}(\Omega)\right)
\end{aligned}
$$

where $\beta=\lambda+2 \mu>0$ (see (2.1.1) and (1.2)). In the sequel we denote by $\langle\cdot, \cdot\rangle$ either the dual pairing between $\left(H_{z}^{2}(\Omega)\right)^{\prime}$ and $H_{z}^{2}(\Omega)$ or the scalar product in $L^{2}(\Omega)$, by $\|\cdot\|$ and $(\cdot, \cdot)$ the norm and the scalar product in $L^{2}(\Omega)$, respectively.

Let the data of the problem satisfy the conditions

$$
\begin{aligned}
& F \in L^{2}(Q), \quad f_{0} \in H^{1}(0, T) \quad(s=0,1) \\
& G \in H^{1}\left(0, T, L^{2}(\Omega)\right) \\
& \theta_{0} \in H^{1}(\Omega), \quad w_{0} \in H_{0}^{1}(\Omega), \quad u_{0} \in H_{z}^{3}(\Omega), \quad\left(\chi_{1,0}, \chi_{2,0}\right) \in \mathbf{K} \\
& F \geq 0 \text { a.c. in } Q, \quad \theta_{0} \geq 0 \text { in } \Omega, \quad f_{s} \geq 0 \text { in }(0, T) \quad(s=0,1)
\end{aligned}
$$


and let the function $\alpha$ be non-negative, vanishing from $\theta_{c}>0$,

$$
\begin{aligned}
& \alpha \in C^{2}(\mathbb{R}), \quad \alpha^{\prime}(\xi)=0 \text { for all } \xi \in \mathbb{R} \backslash\left(0, \theta_{c}\right), \\
& c_{\alpha}=\left\|\alpha^{\prime \prime}\right\|_{L^{\infty}\left(0, \theta_{c}\right)} \quad \text { is sufficiently small. }
\end{aligned}
$$

\section{Problem (P2). Find}

$$
\begin{aligned}
& \theta \in L^{2}\left(0, T, H^{2}(\Omega)\right) \cap H^{1}\left(0, T, L^{2}(\Omega)\right) \cap C^{0}\left(0, T, H^{1}(\Omega)\right), \theta \geq 0 \text { a.e. in } Q \\
& u \in W^{1, \infty}\left(0, T, H_{0}^{1}(\Omega)\right) \cap L^{\infty}\left(0, T, H_{z}^{3}(\Omega)\right) \cap H^{2}\left(0, T,\left(H_{z}^{2}(\Omega)\right)^{\prime}\right) \\
& \chi_{1}, \chi_{2} \in H^{1}\left(0, T, L^{2}(\Omega)\right), \quad\left(\chi_{1}, \chi_{2}\right) \in \mathbf{K}
\end{aligned}
$$

such that

$$
\begin{aligned}
& \partial_{t}\left(c_{0} \theta-l \theta_{*} \chi_{1}\right)+\partial_{t}\left(\left(\alpha(\theta)-\theta \alpha^{\prime}(\theta)\right) \chi_{2} u_{x}\right)-h \theta_{x x}=F+\alpha(\theta) \chi_{2} u_{x t} \\
& \text { a.e. in } Q \\
& (-1)^{s} h \theta_{x}(s, t)+\eta_{s}\left(\theta(s, t)-f_{s}(t)\right)=0 \text { a.e. in }(0, T), \text { for } s=0,1 \\
& \theta(x, 0)=\theta_{0}(x) \text { a.e. in } \Omega \\
& \left\langle u_{t t}, v\right\rangle+a(u, v)+\left(\alpha(\theta) \chi_{2}, v_{x}\right)=(G, v) \text { a.e. in }(0, T), \forall v \in H_{z}^{2}(\Omega) \\
& u(x, 0)=u_{0}(x) \text { and } u_{t}(x, 0)=w_{0}(x) \text { a.e. on } \Omega \\
& \sum_{j=1}^{2} k\left(\partial_{t} \chi_{j}, \chi_{j}-\gamma_{j}\right)+l\left(\theta-\theta_{*}, \chi_{1}-\gamma_{1}\right)+\left(\alpha(\theta) u_{x}, \chi_{2}-\gamma_{2}\right) \leq 0 \\
& \text { a.e. in } Q, \text { for all }\left(\gamma_{1}, \gamma_{2}\right) \in \mathbf{K} \\
& \left(\chi_{1}, \chi_{2}\right)(x, 0)=\left(\chi_{1,0}, \chi_{2,0}\right)(x) \text { a.e. in } \Omega .
\end{aligned}
$$

Remark 3. Formally equation (2.2.6) is equivalent to equation (1.2), where $u$ satisfies the boundary conditions

$$
u(s, t)=u_{x x}(s, t)=0
$$

for $s=0,1$ and $t \in(0, T)$.

Lemma 3 (see N. Chemetov [1]). Under conditions (2.2.2)-(2.2.3) Problem (P2) has at least one solution and for some constant $\mathbf{B}^{\prime}$

$$
\left|u_{x}(x, t)\right| \leq \mathbf{B}^{\prime} \quad \text { for a.e. }(x, t) \in Q \text {. }
$$

Remark 4. The last assumption in (2.2.3) is a compatibility condition with data of Problem (P2). Notice that if one knows that $c_{\alpha}$ is bounded (say $0 \leq c_{\alpha}<1$ ), then it is possible to determine a constant $\mathbf{B}^{\prime}$ depending only on data (2.2.2) (as in the construction of the solution in [1]). And moreover, if $c_{\alpha}$ is sufficiently small in the sense that

$$
0<c_{0}-\theta_{c} c_{\alpha} \mathbf{B}^{\prime}=\mathbf{A},
$$


then we can assure the existence of the solution of problem (P2).

Theorem 2. Under the conditions of Lemma 4 and (2.2.8)-(2.2.9) the solution of Problem (P2) is unique.

Remark 5. Here and in what follows $\mathbf{A}, \mathbf{B}, \mathbf{B}^{\prime}, C_{1}, C_{2}, \ldots>0$ will denote constants that are independent of $x, t$ and, possibly, depend on the data of Problems (P1) and (P2), i.e. on $\theta_{0}, w_{0}, u_{0}, \chi_{1,0}, \chi_{2,0}, \eta, f, F, \mathbf{G}, \mathbf{g}, c_{0}, h, L, \nu, \beta, k, l, \theta_{c}, c_{\alpha}$ and $\theta_{*}$.

\section{Common estimates of the difference of solutions}

In the section we deduce estimates that are true for both problems (P1) and (P2). First let us make few remarks about some usefull notations which we use in what follows. In this section, just for shortness of explanation, formulaes (2.1.1) - (2.1.9) and (2.2.1) (2.2.9) are denoted by (2.J.1) - (2.J.9). Let us suppose that system (2.J.5) - (2.J.7) has two different solutions $\theta^{1}, \mathbf{u}^{1}, \chi_{1}^{1}, \chi_{2}^{1}$ and $\theta^{2}, \mathbf{u}^{2}, \chi_{1}^{2}, \chi_{2}^{2}$, and denote by $\bar{\varphi}$ the difference of two functions $\varphi^{1}$ and $\varphi^{2}$, i.e.

$$
\bar{\varphi}=\varphi^{2}-\varphi^{1} .
$$

Also, in the sequel we often use two trivial identities

$$
\overline{\varphi \psi}=\bar{\varphi} \cdot \psi^{2}+\varphi^{1} \cdot \bar{\psi}=\bar{\varphi} \cdot \psi^{1}+\varphi^{2} \cdot \bar{\psi}
$$

that allow us to have a necessary factor $\psi^{1}$ or $\psi^{2}$ for $\bar{\varphi}$. Therefore without loss of generality we can write this identity omitting the superscripts, i.e.

$$
\overline{\varphi \psi}=\bar{\varphi} \cdot \psi+\varphi \cdot \bar{\psi}
$$

The following estimate plays the crucial role in the proof of two uniqueness results.

Lemma 4. There exists a constant $C_{1}$ such that

$$
\begin{aligned}
& \frac{3 \mathbf{A}}{4} \int_{0}^{t}\|\bar{\theta}\|^{2} d \tau+\frac{h}{2}\|\nabla \hat{\bar{\theta}}\|^{2}(t)+\frac{\eta}{2}\|\hat{\bar{\theta}}\|_{\partial \Omega}^{2}(t) \\
& \quad \leq C_{1} \int_{0}^{t}\left(\sum_{j=1}^{2}\left\|\overline{\chi_{j}}\right\|^{2}(\tau)\right) d \tau+\theta_{c}^{2} c_{\alpha} \int_{0}^{t}\|\bar{\theta}\| \cdot\|\operatorname{div} \overline{\mathbf{u}}\| d \tau+\left|I_{1}\right|+\left|I_{2}\right|+\left|I_{3}\right|
\end{aligned}
$$

where $\hat{\bar{\theta}}(x, t)=\int_{0}^{t} \bar{\theta}(x, s) d s$ and

$$
\begin{aligned}
I_{1}= & \int_{0}^{t} \int_{\Omega} \bar{\theta}(x, \tau)\left[\int_{0}^{\tau} \overline{\chi_{2} \alpha(\theta)} \cdot(\operatorname{div} \mathbf{u})_{s} d s\right] d x d \tau \\
I_{2}= & \int_{\Omega}^{t} \overline{\bar{\theta}}(x, t)\left[\int_{0}^{t}\left(\chi_{2} \alpha(\theta)\right)_{\tau} \cdot \operatorname{div} \overline{\mathbf{u}} d \tau\right] d x \\
& \cdot \int_{0}^{t} \int_{\Omega} \hat{\bar{\theta}} \cdot\left(\chi_{2} \alpha(\theta)\right)_{\tau} \cdot \operatorname{div} \overline{\mathbf{u}} d x d \tau .
\end{aligned}
$$


Proof. Taking the difference of (2.J.5 $)^{1}$ and (2.J.5 $)^{2}$ and integrating it on the time variable over $(0, \tau)$ we have

$$
\begin{aligned}
& \left.\overline{\left(c_{0} \theta-l \theta_{*} \chi_{1}+\left(\alpha(\theta)-\theta \alpha^{\prime}(\theta)\right) \chi_{2} \operatorname{div} \mathbf{u}\right.}, \phi\right)(\tau)+h(\nabla \hat{\bar{\theta}}, \phi)(\tau)+\eta \int_{\partial \Omega} \hat{\bar{\theta}} \phi d x(\tau) \\
& =\left(\int_{0}^{\tau} \frac{}{\alpha(\theta) \chi_{2}(\operatorname{div} \mathbf{u})_{s}} d s, \phi\right) \quad \text { for a.e. } \tau \in(0, T), \forall \phi \in H^{1}(\Omega) .
\end{aligned}
$$

Due to the mean value theorem there is some function $\xi(x, \tau)$ with values between $\theta^{1}(x, \tau)$ and $\theta^{2}(x, \tau)$ such that $\overline{\alpha(\theta)-\theta \alpha^{\prime}(\theta)}=-\xi \alpha^{\prime \prime}(\xi) \cdot \bar{\theta}$ for a.e. $(x, \tau) \in Q$. Hence applying identity (3.1) we get

$$
\begin{aligned}
& \left(\left[c_{0}-\xi \alpha^{\prime \prime}(\xi) \chi_{2} \operatorname{div} \mathbf{u}\right] \cdot \bar{\theta}, \phi\right)+h(\nabla \hat{\bar{\theta}}, \phi)+\eta \int_{\partial \Omega} \hat{\bar{\theta}} \phi d x \\
& \quad=\left(l \theta_{*} \cdot \overline{\chi_{1}}-\left(\alpha(\theta)-\theta \alpha^{\prime}(\theta)\right) \overline{\chi_{2}} \operatorname{div} \mathbf{u}+J+\int_{0}^{\tau} \overline{\alpha(\theta) \chi_{2}}(\operatorname{div} \mathbf{u})_{s} d s, \phi\right)
\end{aligned}
$$

where

$$
\begin{aligned}
J & =-\left(\alpha(\theta)-\theta \alpha^{\prime}(\theta)\right) \chi_{2} \operatorname{div} \overline{\mathbf{u}}+\int_{0}^{\tau} \alpha(\theta) \chi_{2} \overline{(\operatorname{div} \mathbf{u})_{s}} d s \\
& =\theta \alpha^{\prime}(\theta) \chi_{2} \operatorname{div} \overline{\mathbf{u}}-\int_{0}^{\tau}\left(\alpha(\theta) \chi_{2}\right)_{s} \cdot \overline{\operatorname{div} \mathbf{u}} d s .
\end{aligned}
$$

Here we have combined, using integration by parts, the terms in a more convenient form for the following considerations. Therefore, if we substitute in (3.6) $\phi=\bar{\theta}$ and integrate it on $(0, t)$, taking into account (2.J.9) and $\alpha(\theta), \alpha^{\prime}(\theta), \chi_{2} \in L^{\infty}(Q)$, we deduce

$$
\begin{aligned}
\mathbf{A} \int_{0}^{t} & \|\bar{\theta}\|^{2} d t+\frac{h}{2}\|\nabla \hat{\bar{\theta}}\|^{2}(t)+\frac{\eta}{2}\|\hat{\bar{\theta}}\|_{\partial \Omega}^{2}(t) \\
\leq & \int_{0}^{t} \int_{\Omega}\left[c_{0}-\xi \alpha^{\prime \prime}(\xi) \chi_{2} \operatorname{div} \mathbf{u}\right] \cdot|\bar{\theta}|^{2} d x d \tau+\frac{h}{2}\|\nabla \hat{\bar{\theta}}\|^{2}(t)+\frac{\eta}{2}\|\hat{\bar{\theta}}\|_{\partial \Omega}^{2}(t) \\
\leq & C_{2}\left(\int_{0}^{t} \int_{\Omega}|\bar{\theta}|\left|\overline{\chi_{1}}\right| d x d \tau+\int_{0}^{t} \int_{\Omega}|\bar{\theta}|\left|\overline{\chi_{2}}\right| d x d \tau\right) \\
& +\left\|\theta \alpha^{\prime}(\theta)\right\|_{L^{\infty}(Q)}\left\|\chi_{2}\right\|_{L^{\infty}(Q)} \int_{0}^{t} \int_{\mathbf{\Omega}}|\bar{\theta}||\overline{\operatorname{div} \mathbf{u}}| d x d \tau \\
& +\left|\int_{0}^{t} \int_{\Omega}^{\tau} \bar{\theta}(x, \tau)\left(\int_{0}^{\tau}\left(\alpha(\theta) \chi_{2}\right)_{s} \overline{\operatorname{div} \mathbf{u}} d s\right) d x d \tau\right|
\end{aligned}
$$




$$
\begin{aligned}
& +\left|\int_{0}^{t} \int_{\Omega} \bar{\theta}(x, \tau)\left[\int_{0}^{\tau} \overline{\alpha(\theta) \chi_{2}} \cdot(\operatorname{div} \mathbf{u}), d s\right] d x d \tau\right| \\
& =J_{1}+J_{2}+|I|+\left|I_{1}\right| .
\end{aligned}
$$

By the inequality $a b \leq \varepsilon \frac{a^{2}}{2}+\frac{b^{2}}{2 \varepsilon}$,

$$
J_{1} \leq \frac{\mathbf{A}}{4} \int_{0}^{t}\|\bar{\theta}\|^{2} d t+C_{1} \int_{0}^{t}\left(\sum_{j=1}^{2}\left\|\overline{\chi_{j}}\right\|^{2}\right) d \tau
$$

Due to Remark 1,

$$
J_{2} \leq \theta_{c}^{2} \cdot c_{\alpha} \int_{0}^{t}\|\bar{\theta}\|\|\operatorname{div} \overline{\mathbf{u}}\| d \tau .
$$

To conclude the proof of this lemma we need just to rewrite the integral $I$ in (3.8) using the fact that $\bar{\theta}=(\overline{\bar{\theta}})_{t}$ and integration by parts in the time variable:

$$
\begin{aligned}
|I| & =\left|\int_{0}^{t} \int_{\mathbf{\Omega}}(\hat{\bar{\theta}}(x, \tau))_{\tau}\left(\int_{0}^{\tau}\left(\alpha(\theta) \chi_{2}\right)_{s} \overline{\operatorname{div} \mathbf{u}} d s\right) d x d \tau\right| \\
& =\left|\int_{\Omega} \hat{\bar{\theta}}(x, t)\left(\int_{0}^{t}\left(\alpha(\theta) \chi_{2}\right)_{s} \overline{\operatorname{div} \mathbf{u}} d s\right) d x-\int_{0}^{t} \int_{\Omega} \hat{\bar{\theta}}\left(\alpha(\theta) \chi_{2}\right)_{s} \overline{\operatorname{div} \mathbf{u}} d x d \tau\right| \\
& =\left|I_{2}-I_{3}\right| \leq\left|I_{2}\right|+\left|I_{3}\right| .
\end{aligned}
$$

The lemma is proved

Lemma 5. There exists a constant $C_{3}$ such that

$$
\sum_{j=1}^{2} k\left\|\overline{\chi_{j}}\right\|^{2}(t) \leq C_{3}\left(\int_{0}^{t}\|\bar{\theta}\|^{2} d \tau+\int_{0}^{t}\|\operatorname{div} \overline{\mathbf{u}}\|^{2} d \tau\right) .
$$

Proof. To show estimate (3.9), we choose $\left(\gamma_{1}, \gamma_{2}\right)=\left(\chi_{1}^{2}, \chi_{2}^{2}\right)$ in equation (2.J.7 $)^{1}$ and $\left(\gamma_{1}, \gamma_{2}\right)=\left(\chi_{1}^{1}, \chi_{2}^{1}\right)$ in equation (2.J.7 $)^{2}$. Taking the sum of the deduced inequalities and integrating it on the spatial variable $x \in \Omega$ and the time variable in $(0, t)$ we easily get

$$
\sum_{j=1}^{2} \frac{k}{2}\left\|\overline{\chi_{j}}\right\|^{2}(t)+\int_{0}^{\ell} \int_{\Omega}\left[l \cdot \bar{\theta} \cdot \overline{\chi_{1}}+\overline{\alpha(\theta) \operatorname{div} \mathbf{u}} \cdot \overline{\chi_{2}}\right] d \tau d x \leq 0 .
$$

Hence using that $\alpha(\theta), \alpha^{\prime}(\theta)$, divu $\in L^{\infty}(Q)$ (see (2.J.8)) in the relation

$$
\overline{\alpha(\theta) \operatorname{div} \mathbf{u}}=\overline{\alpha(\theta)} \cdot \operatorname{div} \mathbf{u}+\alpha(\theta) \overline{\operatorname{div} \mathbf{u}}=\alpha^{\prime}(\xi) \cdot \bar{\theta}: \operatorname{div} \mathbf{u}+\alpha(\theta) \overline{\operatorname{div} u}
$$


for some $\xi$ with values between $\theta^{1}, \theta^{2}$ and applying the inequality $a b \leq \varepsilon \frac{a^{2}}{2}+\frac{b^{2}}{2 \varepsilon}$ in the last integral of (3.10) we obtain

$$
\sum_{j=1}^{2} \frac{k}{2}\left\|\overline{\chi_{j}}\right\|^{2}(t) \leq \int_{0}^{t}\left(\sum_{j=1}^{2} \frac{k}{2}\left\|\overline{\chi_{j}}\right\|^{2}(\tau)\right) d \tau+C_{4} \int_{0}^{t}\left(\|\bar{\theta}\|^{2}+\|\operatorname{div} \overline{\mathbf{u}}\|^{2}\right) d \tau
$$

Therefore, due to the Gronwall inequality we deduce the desirable inequality (3.9)

\section{Uniqueness result for Problem (P1)}

In order to get this uniqueness result, first we present an auxiliary lemma.

Lemma 6. There exists a constant $C_{5}$ such that

$$
\begin{aligned}
& \nu \int_{0}^{t}\|\nabla(\operatorname{div} \overline{\mathbf{u}})\|^{2} d \tau+\frac{7}{8}\left(\lambda+\frac{2}{3} \mu\right) \int_{0}^{t}\|\operatorname{div} \overline{\mathbf{u}}\|^{2} d \tau \\
& \leq C_{5} \int_{0}^{t}\left\|\overline{\chi_{2}}\right\|^{2} d \tau+\theta_{c} c_{\alpha} \int_{0}^{t}\|\bar{\theta}\| \cdot\|\operatorname{div} \overline{\mathbf{u}}\| d \tau
\end{aligned}
$$

Proof. By the momentum equation (2.J.6) for $\overline{\mathbf{u}}$ we have

$$
a(\overline{\mathbf{u}}, \overline{\mathbf{u}})+\left(\overline{\alpha(\theta) \chi_{2}}, \operatorname{div} \overline{\mathbf{u}}\right)=0 \quad \text { a.e. in }(0, T) .
$$

Due to $\alpha(\theta), \alpha^{\prime}(\theta), \chi_{2} \in L^{\infty}(Q)$, Remark 1 and the inequality $a b \leq \varepsilon \frac{a^{2}}{2}+\frac{b^{2}}{2 \varepsilon}$,

$$
\begin{aligned}
\nu \| \nabla & (\operatorname{div} \overline{\mathbf{u}})\left\|^{2}+\left(\lambda+\frac{2}{3} \mu\right)\right\| \operatorname{div} \overline{\mathbf{u}} \|^{2} \\
& \leq|a(\overline{\mathbf{u}}, \overline{\mathbf{u}})| \\
& \leq\left|\int_{\mathbf{\Omega}} \overline{\alpha(\theta) \chi_{2}} \cdot \operatorname{div} \overline{\mathbf{u}} d x\right| \\
& \leq\left|\int_{\Omega} \alpha(\theta) \cdot \overline{\chi_{2}} \operatorname{div} \overline{\mathbf{u}} d x\right|+\left|\int_{\Omega} \alpha^{\prime}(\xi) \chi_{2} \cdot \bar{\theta} \cdot \operatorname{div} \overline{\mathbf{u}} d x\right| \\
& \leq C_{5}\left\|\overline{\chi_{2}}\right\|^{2}+\frac{1}{8}\left(\lambda+\frac{2}{3} \mu\right)\|\operatorname{div} \overline{\mathbf{u}}\|^{2}+\theta_{c} c_{\alpha}\|\bar{\theta}\| \cdot\|\operatorname{div} \overline{\mathbf{u}}\| .
\end{aligned}
$$

Hence integrating this inequality in the time variable over the interval $[0, t]$ we obtain (4.1) 
Proof of Theorem 1. Taking the sum of (3.2) and (4.1), and then applying (2.1.10) in the inequality

$$
\theta_{c}\left(\theta_{c}+1\right) c_{\alpha}\|\bar{\theta}\| \cdot\|\operatorname{div} \overline{\mathbf{u}}\| \leq \frac{\mathbf{A}}{2}\|\bar{\theta}\|^{2}+\frac{1}{2}\left(\lambda+\frac{2}{3} \mu\right)\|\operatorname{div} \overline{\mathbf{u}}\|^{2}
$$

we show that.

$$
\begin{aligned}
& \frac{\mathbf{A}}{4} \int_{0}^{t}\|\bar{\theta}\|^{2} d \tau+\frac{h}{2}\|\nabla \hat{\bar{\theta}}\|^{2}(t)+\frac{\eta}{2}\|\hat{\bar{\theta}}\|_{\partial \Omega}^{2}(t) \\
& \quad+\nu \int_{0}^{t}\|\nabla(\operatorname{div} \overline{\mathbf{u}})\|^{2} d \tau+\frac{3}{8}\left(\lambda+\frac{2}{3} \mu\right) \int_{0}^{t}\|\operatorname{div} \overline{\mathbf{u}}\|^{2} d \tau \\
& \leq C_{6} \int_{0}^{t}\left(\sum_{j=1}^{2}\left\|\overline{\chi_{j}}\right\|^{2}(\tau)\right) d \tau+\left|I_{1}\right|+\left|I_{2}\right|+\left|I_{3}\right| .
\end{aligned}
$$

In order to estimate the integral $I_{1}$, first we show that divu $\mathbf{u}_{t} \in L^{2}\left(0, T, C^{0}(\bar{\Omega})\right)$. In fact, due to (2.1.4) the function

$$
\phi(t)=\left(\left\|\nabla\left(\operatorname{div} \mathbf{u}_{t}\right)\right\|^{2}+\left\|\left(\operatorname{div} \mathbf{u}_{t}\right)\right\|^{2}+\sum_{i=1}^{3}\left\|\nabla\left(u_{i}\right)_{t}\right\|^{2}+\left\|\left(\alpha(\theta) \chi_{2}\right)_{t}\right\|^{2}\right)(t)
$$

is such that

$$
\phi(t) \in L^{1}(0, T) \quad \text { and, of course, } \quad \phi(t)<\infty \text { for a.e. } t \in(0, T) .
$$

Hence from (2.1.6) it follows that the function $\mathbf{u}_{\imath}$ satisfies for a.e. $t \in(0, T)$

$$
\begin{gathered}
\frac{\partial}{\partial x_{i}} P=\mathbf{G}_{t}-\mu \operatorname{div}\left(\nabla\left(u_{i}\right)_{t}\right)-\frac{\partial}{\partial x_{i}}\left(\left(\alpha(\theta) \chi_{2}\right)_{t}\right) \quad \text { in } D^{\prime}(\Omega) \quad(i=1,2,3) \\
P=(\lambda+\mu) \operatorname{div} \mathbf{u}_{t}-\nu \Delta\left(\operatorname{div} \mathbf{u}_{t}\right)
\end{gathered}
$$

and three boundary conditions in a suitable sense which are similar to the conditionsof Remark 2. This identity and (4.3) imply that $P(x, t), P_{x_{i}}(x, t) \in H^{-1}(\Omega)$ for a.e. $t \in(0, T)$. From [6: Theorem 3.2] (see also [3: Proof of Lemma 1]) it follows $P \in L^{2}(\Omega)$ and

$$
\|P\|(t) \leq C_{7}\left(\|P\|_{H^{-1}(\Omega)}+\left\|P_{x_{i}}\right\|_{H^{-1}(\Omega)}\right)(t) .
$$

Hence by regularity results for elliptic boundary value problems

$$
\left\|\operatorname{div} \mathbf{u}_{t}\right\|_{C^{\circ}(\bar{\Omega})}(t) \leq C_{7}\left\|\Delta \operatorname{div} \mathbf{u}_{t}\right\|(t)
$$

and by (4.3), (4.4) we deduce

$$
\int_{0}^{T}\left\|\operatorname{div} \mathbf{u}_{t}\right\|_{C^{0}(\tilde{\Omega})}^{2} d t<\infty
$$


Therefore, by $\alpha(\theta), \alpha^{\prime}(\theta), \chi_{2} \in L^{\infty}(Q)$ and $a b \leq \varepsilon \frac{a^{2}}{2}+\frac{b^{2}}{2 \varepsilon}$ we have

$$
\begin{aligned}
\left|I_{1}\right| & \leq \int_{0}^{t} \int_{0}^{\tau}\left\|(\operatorname{div} \mathbf{u})_{t}\right\|_{C^{0}(\bar{\Omega})}(s)\left(\int_{\Omega}|\bar{\theta}|(x, \tau) \cdot\left|\overline{\alpha(\theta) \chi_{2}}\right|(x, s) d x\right) d s d \tau \\
& \leq \int_{0}^{t}\|\bar{\theta}\|(\tau)\left(\int_{0}^{\tau}\left\|(\operatorname{div} \mathbf{u})_{\ell}\right\|_{C^{\circ}(\bar{\Omega})}(s) \cdot\left\|\overline{\alpha(\theta) \chi_{2}}\right\|(s) d s\right) d \tau \\
& \leq \frac{A}{8} \int_{0}^{t}\|\bar{\theta}\|^{2} d \tau+\frac{1}{A} \int_{0}^{t}\left(\int_{0}^{t}\left\|(\operatorname{div} \mathbf{u})_{t}\right\|_{C^{\circ}(\bar{\Omega})}(s) \cdot\left\|\overline{\alpha(\theta) \chi_{2}}\right\|(s) d s\right)^{2} d \tau \\
& \leq \frac{A}{8} \int_{0}^{t}\|\bar{\theta}\|^{2} d \tau+C_{8}\left\{t \int_{0}^{t}\left\|(\operatorname{div} \mathbf{u})_{t}\right\|_{C^{0}(\bar{\Omega})}^{2} d \tau\right\} \cdot \int_{0}^{t}\left(\left\|\overline{\chi_{2}}\right\|^{2}+\|\bar{\theta}\|^{2}\right) d \tau .
\end{aligned}
$$

By the Hölder inequality and the embedding theorem $H^{1}(\Omega) \subset L^{4}(\Omega)$, we get

$$
\begin{aligned}
\left|I_{2}\right| \leq & \|\hat{\bar{\theta}}\|_{L^{4}(\Omega)}(t) \cdot \int_{0}^{t}\|\operatorname{div} \overline{\mathbf{u}}\|_{L^{4}(\Omega)}\left\|\left(\alpha(\theta) \chi_{2}\right)_{t}\right\| d \tau \\
\leq & C_{9}\|\hat{\bar{\theta}}\|_{H^{2}(\Omega)}(t) \cdot \int_{0}^{t}\|\operatorname{div} \overline{\mathbf{u}}\|_{H^{1}(\Omega)}\left\|\left(\alpha(\theta) \chi_{2}\right)_{t}\right\| d \tau \\
\leq & \frac{h}{4}\|\nabla \hat{\bar{\theta}}\|^{2}(t)+\frac{\eta}{4}\|\hat{\bar{\theta}}\|_{\partial \Omega}^{2}(t)+C_{10} \int_{0}^{t}\left\|\left(\alpha(\theta) \chi_{2}\right)_{t}\right\|^{2} d \tau \\
& \times \int_{0}^{t}\left(\nu\|\nabla(\operatorname{div} \overline{\mathbf{u}})\|^{2}+\left(\lambda+\frac{2}{3} \mu\right)\|\operatorname{div} \overline{\mathbf{u}}\|^{2}\right) d \tau .
\end{aligned}
$$

Let us apply the same idea to estimate the integral $I_{3}$ :

$$
\begin{aligned}
\left|I_{3}\right| \leq & \int_{0}^{t}\|\overline{\bar{\theta}}\|_{L^{4}(\Omega)} \cdot\|\operatorname{div} \overline{\mathbf{u}}\|_{L^{4}(\Omega)} \cdot\left\|\left(\alpha(\theta) \chi_{2}\right)_{t}\right\| d \tau \\
\leq & C_{11} \int_{0}^{t}\|\hat{\bar{\theta}}\|_{H^{1}(\Omega)} \cdot\|\operatorname{div} \overline{\mathbf{u}}\|_{H^{1}(\Omega)} \cdot\left\|\left(\alpha(\theta) \chi_{2}\right)_{t}\right\| d \tau \\
\leq & C_{12} \int_{0}^{t}\left\|\left(\alpha(\theta) \chi_{2}\right)_{t}\right\|^{2}\left(\frac{h}{4}\|\nabla \hat{\bar{\theta}}\|^{2}+\frac{\eta}{4}\|\hat{\bar{\theta}}\|_{\gamma}^{2}\right) d \tau \\
& +\int_{0}^{t}\left(\frac{7}{8} \nu\|\nabla(\operatorname{div} \overline{\mathbf{u}})\|^{2}+\frac{1}{4}\left(\lambda+\frac{2}{3} \mu\right)\|\operatorname{div} \overline{\mathbf{u}}\|^{2}\right) d \tau .
\end{aligned}
$$


Substituting estimates (4.5) - (4.7) into (4.2) we deduce

$$
\begin{aligned}
\frac{\mathbf{A}}{8} \int_{0}^{t}\|\bar{\theta}\|^{2} d \tau+\frac{h}{4}\|\nabla \hat{\bar{\theta}}\|^{2}(t)+\frac{\eta}{4}\|\hat{\bar{\theta}}\|_{\partial \Omega}^{2}(t) \\
\quad+\frac{1}{8} \int_{0}^{t}\left(\nu\|\nabla(\operatorname{div} \overline{\mathbf{u}})\|^{2}+\left(\lambda+\frac{2}{3} \mu\right)\|\operatorname{div} \overline{\mathbf{u}}\|^{2}\right) d \tau \\
\leq C_{8}\left(t \int_{0}^{t}\left\|(\operatorname{div} \mathbf{u})_{t}\right\|_{C^{0}(\bar{\Omega})}^{2} d \tau\right) \cdot \int_{0}^{t}\|\bar{\theta}\|^{2} d \tau \\
+C_{12} \int_{0}^{t}\left\|\left(\alpha(\theta)_{\chi_{2}}\right)_{t}\right\|^{2} \cdot\left(\frac{h}{4}\left\|\nabla \overline{\bar{\theta}}^{2}+\frac{\eta}{4}\right\| \overline{\bar{\theta}} \|_{\partial \Omega}^{2}\right) d \tau \\
+C_{10} \int_{0}^{t}\left\|\left(\alpha(\theta)_{\chi_{2}}\right)_{t}\right\|^{2} d \tau \cdot \int_{0}^{t}\left(\nu\|\nabla(\operatorname{div} \mathbf{u})\|^{2}+\left(\lambda+\frac{2}{3} \mu\right)\|\operatorname{div} \mathbf{u}\|^{2}\right) d \tau \\
\left.\left.+\max ^{t} C_{6}, C_{8} t \int_{0}^{t}\left\|(\operatorname{div} \mathbf{u})_{\ell}\right\|_{C^{0}(\bar{\Omega})}^{2} d \tau\right)\right\} \cdot \int_{0}^{t}\left(\sum_{j=1}^{2}\left\|\overline{\chi_{j}}\right\|^{2}(\tau)\right) d \tau .
\end{aligned}
$$

Let us choose $\bar{t}$ such that

$$
C_{8} \bar{t} \int_{0}^{\bar{t}}\left\|(\operatorname{div} \mathbf{u})_{t}\right\|_{C^{\circ}(\bar{\Omega})}^{2} d \tau \leq \frac{A}{16} \quad \text { and } \quad C_{10} \int_{0}^{\bar{t}}\left\|\left(\alpha(\theta) \chi_{2}\right)_{t}\right\|^{2} d \tau \leq \frac{1}{16}
$$

and denote

$$
\begin{aligned}
y(t)= & \frac{\mathbf{A}}{16} \int_{0}^{t}\|\bar{\theta}\|^{2} d \tau+\frac{h}{4}\|\nabla \hat{\bar{\theta}}\|^{2}(t)+\frac{\eta}{4}\|\hat{\bar{\theta}}\|_{\partial \Omega}^{2}(t) \\
& +\frac{1}{16} \int_{0}^{t}\left(\nu\|\nabla(\operatorname{div} \overline{\mathbf{u}})\|^{2}+\left(\lambda+\frac{2}{3} \mu\right)\|\operatorname{div} \overline{\mathbf{u}}\|^{2}\right) d \tau
\end{aligned}
$$

Then applying estimate (3.9) from (4.8) we easily get that $y(t)$ satisfies the Gronwall inequality

$$
y(t) \leq \int_{0}^{t} G(\tau) y(\tau) d \tau \quad(0 \leq t \leq \bar{t})
$$

where

$$
G(t)=\max _{0<t<i}\left(C_{13}, C_{12}\left\|\left(\alpha(\theta) \chi_{2}\right)_{t}\right\|^{2}(t)\right) \in L^{1}(0, \bar{t}) .
$$

Hence $y(t)=0$ or $\bar{\theta}=0, \overline{\mathbf{u}}=0, \bar{\chi}_{1}=0, \bar{\chi}_{2}=0$ for any $0 \leq t \leq \bar{t}$.

We can repeat the same estimates for the interval $[\bar{t}, 2 \bar{t}]$ and so on. Therefore the solution of Problem (P1) is unique 


\section{Uniqueness result for Problem (P2)}

In this section $\theta^{1}, u^{1}, \chi_{1}^{1}, \chi_{2}^{1}$ and $\theta^{2}, u^{2}, \chi_{1}^{2}, \chi_{2}^{2}$ are two different solutions of Problem (P2) and $\bar{\theta}, \bar{u}, \bar{\chi}_{1}, \bar{\chi}_{2}$ is their difference.

Lemma 7. There exists a constant $C_{14}$ such that

$$
\begin{aligned}
& \frac{1}{2}\left[\left\|\bar{u}_{x}\right\|^{2}+\beta\|\| \hat{\bar{u}}_{x x} \|^{2}\right](t)+\frac{\nu}{4}\left\|\hat{\bar{u}}_{x x x}\right\|^{2}(t) \\
& \quad \leq C_{14} \int_{0}^{t}\left\|\hat{\bar{u}}_{x x x}\right\|^{2} d \tau+\left(\frac{\mathbf{A}}{4}+C_{14} t\right) \int_{0}^{t}\|\bar{\theta}\|^{2} d \tau+C_{14} \int_{0}^{t}\left\|\overline{\chi_{2}}\right\|^{2} d \tau .
\end{aligned}
$$

Proof. From (2.2.6) for $\bar{u}$ we have

$$
\left\langle\bar{u}_{t t}, v\right\rangle+a(\bar{u}, v)+\left(\overline{\alpha(\theta) \chi_{2}}, v_{x}\right)=0 \quad \text { a.e. in }(0, T), \text { for all } v \in H_{z}^{2}(\Omega) .
$$

So integrating it in the time variable over the interval $(0, \tau)$ we get

$$
\left\langle\bar{u}_{t}(\tau), v\right\rangle+a(\hat{\bar{u}}(\tau), v)+\left(\int_{0}^{\tau} \overline{\alpha(\theta) \chi_{2}} d s, v_{x}\right)=0 \quad \text { a.e. in }(0, T)
$$

where $\hat{\bar{u}}(\tau)=\int_{0}^{\tau} \bar{u}(s) d s$. Hence taking $v=-\bar{u}_{x x}$ (a rigorous proof that we can use $v=-\bar{u}_{x x}$ as a test function was shown in [1: Page 169/Formulaes (49) and (50)]), integrating on $(0, t)$ we easily deduce

$$
\begin{aligned}
\frac{1}{2}\left[\left\|\bar{u}_{x}\right\|^{2}\right. & \left.+\beta\left\|\hat{\bar{u}}_{x x}\right\|^{2}+\nu\left\|\hat{\bar{u}}_{x x x}\right\|^{2}\right](t) \\
& =\int_{0}^{t} \int_{\Omega}\left(\int_{0}^{\tau} \overline{\alpha(\theta) \chi_{2}} d s\right) \cdot \bar{u}_{x x x} d x d \tau \\
& =\int_{0}^{t} \int_{\Omega}\left(\int_{0}^{\tau} \overline{\alpha(\theta) \chi_{2}} d s\right) \cdot\left(\hat{\bar{u}}_{x x x}\right)_{\tau} d x d \tau \\
& =\int_{\Omega}\left(\int_{0}^{t} \overline{\alpha(\theta) \chi_{2}} d s\right) \cdot \hat{\bar{u}}_{x x x}(t) d x-\int_{0}^{t} \int_{\Omega} \overline{\alpha(\theta) \chi_{2}} \cdot \hat{\bar{u}}_{x x x} d x d \tau \\
& =J_{3}+J_{4} .
\end{aligned}
$$

Due to the inequality $a b \leq \varepsilon \frac{a^{2}}{2}+\frac{b^{2}}{2 \varepsilon}$,

$$
\begin{aligned}
\left|J_{3}\right| & \leq C_{15} \int_{\Omega}\left(\int_{0}^{t} \overline{\alpha(\theta) \chi_{2}} d s\right)^{2} d x+\frac{\nu}{4}\left\|\hat{\bar{u}}_{x x x}\right\|^{2}(t) \\
& \leq C_{16} t \int_{0}^{t}\|\bar{\theta}\|^{2}(\tau) d \tau+C_{16} t \int_{0}^{t}\left\|\overline{\chi_{2}}\right\|^{2}(r) d \tau+\frac{\nu}{4}\left\|\hat{\bar{u}}_{x x x}\right\|^{2}(t)
\end{aligned}
$$


where we have applied (3.11) and $\alpha(\theta), \alpha^{\prime}(\theta), \chi_{2} \in L^{\infty}(Q)$. By the same way

$$
\begin{aligned}
\left|J_{4}\right| & \leq \varepsilon \int_{0}^{t} \int_{\Omega}\left(\overline{\alpha(\theta) \chi_{2}}\right)^{2} d x d \tau+\frac{1}{4 \varepsilon} \int_{0}^{t} \int_{\Omega}\left|\hat{\bar{u}}_{x x x}\right|^{2} d x d \tau \\
& \leq \frac{\mathbf{A}}{4} \int_{0}^{t}\|\bar{\theta}\|^{2} d \tau+C_{17} \int_{0}^{t}\left\|\overline{\chi_{2}}\right\|^{2} d \tau+C_{17} \int_{0}^{t}\left\|\hat{\bar{u}}_{x x x}\right\|^{2} d \tau .
\end{aligned}
$$

Combining all these estimates we obtain inequality (5.1)

Proof of Theorem 2. Let us take the sum of (3.2) and (5.1):

$$
\begin{aligned}
\left(\frac{\mathbf{A}}{2}-\right. & \left.C_{14} t\right) \int_{0}^{t}\|\bar{\theta}\|^{2} d \tau+\frac{h}{2}\left\|\overline{\bar{\theta}}_{x}\right\|^{2}(t) \\
& +\sum_{s=0}^{1} \frac{\eta_{s}}{2}|\overline{\bar{\theta}}(s)|^{2}(t)+\frac{1}{2}\left\|\bar{u}_{x}\right\|^{2}(t)+\frac{\beta}{2}\left\|\hat{\bar{u}}_{x x}\right\|^{2}(t)+\frac{\nu}{4}\left\|\hat{\bar{u}}_{x x x}\right\|^{2}(t) \\
\leq & C_{14} \int_{0}^{t}\left\|\hat{\bar{u}}_{x x x}\right\|^{2} d \tau+C_{1} \int_{0}^{t}\left(\sum_{j=1}^{2}\left\|\overline{\chi_{j}}\right\|^{2}(\tau)\right) d \tau+\left|I_{1}\right|+\left|I_{2}\right|+\left|I_{3}\right| .
\end{aligned}
$$

To estimate the integral $I_{1}$, let us rewrite it using integration by parts:

$$
\begin{aligned}
\left|I_{1}\right| & =\left|\int_{0}^{t} \int_{\Omega}\left(\int_{0}^{\tau} \overline{\chi_{2} \alpha(\theta)} \cdot u_{x t} d s\right) \cdot(\overline{\bar{\theta}})_{\tau} d x d \tau\right| \\
& =\left|\int_{\Omega}\left(\int_{0}^{t} \overline{\chi_{2} \alpha(\theta)} \cdot u_{x t} d s\right) \cdot \hat{\bar{\theta}}(t) d x-\int_{0}^{t} \int_{\Omega} \overline{\chi_{2} \alpha(\theta)} \cdot u_{x t} \cdot \hat{\bar{\theta}} d x d \tau\right| .
\end{aligned}
$$

Therefore, by (2.2.4), the embedding theorem $H^{1}(0,1) \subset C(0,1)$ and the inequality $a b \leq \varepsilon \frac{a^{2}}{2}+\frac{b^{2}}{2 \varepsilon}$,

$$
\begin{aligned}
\left|I_{1}\right| \leq & \|\hat{\bar{\theta}}\|_{C(\Omega)}(t)\left(\int_{0}^{t} \int_{\Omega}\left|u_{x t}\right| \cdot\left|\overline{\alpha(\theta) \chi_{2}}\right| d x d \tau\right) \\
& +\int_{0}^{t}\|\hat{\bar{\theta}}\|_{C(\Omega)}(\tau)\left(\int_{\Omega}\left|u_{x t}\right| \cdot\left|\overline{\alpha(\theta) \chi_{2}}\right| d x\right) d \tau \\
\leq & \frac{h}{8}\left\|\hat{\bar{\theta}}_{x}\right\|^{2}(t)+\sum_{s=0}^{1} \frac{\eta_{s}}{8}|\hat{\bar{\theta}}(s)|^{2}(t) \\
& +\left(C_{18} \int_{0}^{t}\left\|u_{x t}\right\|^{2} d \tau+\frac{\mathbf{A}}{4}\right) \cdot \int_{0}^{t}\left(\left\|\bar{\chi}_{2}\right\|^{2}+\|\bar{\theta}\|^{2}\right) d \tau
\end{aligned}
$$




$$
+C_{18} \int_{0}^{t}\left\|u_{x t}\right\|^{2}\left(\frac{h}{4}\left\|\hat{\bar{\theta}}_{x}\right\|^{2}+\sum_{s=0}^{1} \frac{\eta_{s}}{4}|\hat{\bar{\theta}}(s)|^{2}\right) d \tau
$$

and

$$
\begin{aligned}
\left|I_{2}\right| & \leq\|\hat{\bar{\theta}}\|_{C(\Omega)}(t) \cdot \int_{0}^{t}\left\|\bar{u}_{x}\right\|(\tau) \cdot\left\|\left(\alpha(\theta) \chi_{2}\right)_{t}\right\|(\tau) d \tau \\
& \leq \frac{h}{8}\left\|\hat{\bar{\theta}}_{x}\right\|^{2}(t)+\sum_{s=0}^{1} \frac{\eta_{s}}{8}|\hat{\bar{\theta}}(s)|^{2}(t)+C_{19} \int_{0}^{t}\left\|\left(\alpha(\theta) \chi_{2}\right)_{\imath}\right\|^{2} d \tau \cdot \int_{0}^{t}\left\|\bar{u}_{x}\right\|^{2} d \tau .
\end{aligned}
$$

Applying the same idea to $I_{3}$ we get

$$
\begin{aligned}
\left|I_{3}\right| & \leq \int_{0}^{t}\|\hat{\bar{\theta}}\|_{C(\Omega)} \cdot\left\|\vec{u}_{x}\right\| \cdot\left\|\left(\alpha(\theta) \chi_{2}\right)_{t}\right\| d \tau \\
& \leq C_{20} \int_{0}^{t}\left\|\left(\alpha(\theta) \chi_{2}\right)_{t}\right\|^{2}\left(\frac{h}{4}\left\|\hat{\bar{\theta}}_{x}\right\|^{2}+\sum_{s=0}^{1} \frac{\eta_{s}}{4}|\hat{\bar{\theta}}(s)|^{2}\right) d \tau+\frac{1}{4} \int_{0}^{t}\left\|\bar{u}_{x}\right\|^{2} d \tau .
\end{aligned}
$$

Let us substitute (5.3) - (5.5) into (5.2). Then

$$
\begin{aligned}
\left(\frac{\mathbf{A}}{2}-\right. & \left.C_{14} t-C_{18} \int_{0}^{t}\left\|u_{x t}\right\|^{2} d \tau\right) \cdot \int_{0}^{t}\|\overline{\hat{\theta}}\|^{2} d \tau+\frac{h}{4}\left\|\hat{\bar{\theta}}_{x}\right\|^{2}(t) \\
& +\sum_{s=0}^{1} \frac{\eta_{s}}{4}|\overline{\bar{\theta}}(s)|^{2}(t)+\frac{1}{4}\left\|\bar{u}_{x}\right\|^{2}(t)+\frac{\beta}{2}\left\|\hat{\bar{u}}_{x x}\right\|^{2}(t)+\frac{\nu}{4}\left\|\dot{\overline{\bar{u}}}_{x x x}\right\|^{2}(t) \\
\leq & \int_{0}^{t}\left\{C_{20}\left\|\left(\alpha(\theta) \chi_{2}\right)_{\imath}\right\|^{2}+C_{18}\left\|u_{x t}\right\|^{2}\right\}\left(\frac{h}{4}\left\|\hat{\bar{\theta}}_{x}\right\|^{2}+\sum_{s=0}^{1} \frac{\eta s}{4}|\hat{\bar{\theta}}(s)|^{2}\right) d \tau \\
& +C_{19} \int_{0}^{t}\left\|\left(\alpha(\theta) \chi_{2}\right)_{\imath}\right\|^{2} d \tau \cdot \int_{0}^{t}\left\|\bar{u}_{x}\right\|^{2} d \tau+C_{14} \int_{0}^{t}\left\|\hat{\bar{u}}_{x x x}\right\|^{2} d \tau \\
& +\max \left\{C_{1}, C_{18} \int_{0}^{t}\left\|u_{x t}\right\|^{2} d \tau+\frac{\mathbf{A}}{4}\right\} \cdot \int_{0}^{t}\left(\sum_{j=1}^{2}\left\|\bar{\chi}_{j}\right\|^{2}\right) d \tau .
\end{aligned}
$$

Hence if we define $\bar{t}$ such that

$$
C_{14} \cdot \bar{t}+C_{18} \int_{0}^{\bar{t}}\left\|u_{x t}\right\|^{2} d \tau \leq \frac{A}{4}
$$


and take

$$
y(t)=\frac{\mathbf{A}}{4} \int_{0}^{t}\|\bar{\theta}\|^{2} d \tau+\frac{h}{4}\left\|\hat{\bar{\theta}}_{x}\right\|^{2}(t)+\sum_{s=0}^{1} \frac{\eta g}{4}|\hat{\bar{\theta}}(s)|^{2}(t)+\frac{1}{8}\left\|\bar{u}_{x}\right\|^{2}(t)+\frac{\nu}{4}\left\|\hat{\bar{u}}_{x x x}\right\|^{2}(t),
$$

then from (5.6) and (3.9) we easily deduce that $y(t)$ satisfy to the Gronwall inequality

$$
y(t) \leq \int_{0}^{t} G(\tau) y(\tau) d \tau \quad \text { for any } 0 \leq t \leq \bar{t}
$$

and for some function $G \in L^{1}(0, \bar{t})$. Therefore $y(t)=0$ or $\bar{\theta}=0, \bar{u}=0, \bar{\chi}_{1}=0, \bar{\chi}_{2}=0$ for any $0 \leq t \leq \bar{t}$. Repeating the same estimates for the interval $[\bar{t}, 2 \bar{t}]$ and so on we conclude that the solution for Problem (P2) is unique

Acknowledgement. The autor is deeply indebted to the editors and anonymous referees whose questions and remarks have motivated him to improve the exposition.

\section{References}

[1] Chemetov, N.: Existence result for the full one-dimensional Frémond model of shape memory alloys. Adv. Math. Sci. Appl., Gakkötosho 8 (1998), 157 - 172.

[2] Colli, P.: Global existence for the three-dimensional Frémond model of shape memory alloys. Nonlin. Anal.: Theory, Meth. \& Appl. 24 (1995), 1565 - 1579.

[3] Colli, P., Frémond, M. and A. Visintin: Thermo-mechanical evolution of shape memory alloys. Quart. Appl. Math. 48 (1990), $31-47$.

[4] Colli, P. and J. Sprekels: Global solution to the full one-dimensional Frémond model for shape memory alloys. Math. Meth. Appl. Sci. 18 (1995), $371-385$.

[5] Colli, P. J. and Sprekels: Positivity of temperature in the general Frémond model for shape memory alloys. Contin. Mech. Thermodyn. 5 (1993), $255-264$.

[6] Duvaut, G. and J.-L. Lions: Les inéquations en mécanique et en physique. Paris: Dunod 1972.

[7] Frémond, M.: Matériaux à mémoire de forme. C.R. Acad. Sci. Paris, Sér. II Méc. Phys. Chim. Sci. Univers Sci. Terre 304 (1987), 239 - 244. 